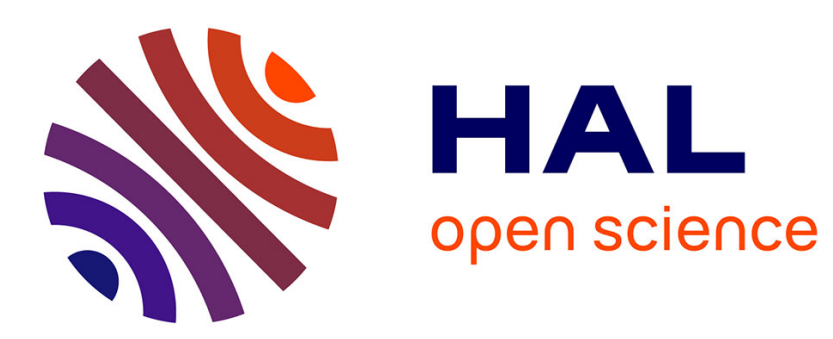

\title{
Anomalous elasticity of an ordered lamellar liquid foam
} Jacques Leng, Frédéric Nallet, Didier Roux

\section{To cite this version:}

Jacques Leng, Frédéric Nallet, Didier Roux. Anomalous elasticity of an ordered lamellar liquid foam. European Physical Journal E: Soft matter and biological physics, 2001, 4, pp.337. hal-00097163

\section{HAL Id: hal-00097163 https://hal.science/hal-00097163}

Submitted on 21 Sep 2006

HAL is a multi-disciplinary open access archive for the deposit and dissemination of scientific research documents, whether they are published or not. The documents may come from teaching and research institutions in France or abroad, or from public or private research centers.
L'archive ouverte pluridisciplinaire HAL, est destinée au dépôt et à la diffusion de documents scientifiques de niveau recherche, publiés ou non, émanant des établissements d'enseignement et de recherche français ou étrangers, des laboratoires publics ou privés. 


\title{
Anomalous elasticity of an ordered lamellar liquid foam
}

\author{
J. Lenga ${ }^{\mathrm{a}}$, F. Nallet, and D. Roux \\ Centre de Recherche Paul-Pascal, CNRS, Avenue du Docteur Schweitzer, F-33600 Pessac, France
}

Received 7 August 2000

\begin{abstract}
We investigate experimentally the linear viscoelastic properties of a lamellar liquid foam as a function of the cell size and spatial organisation. The system consists of multilamellar vesicles generated by a simple shear flow on a lyotropic lamellar phase. The vesicles can be prepared either in an amorphous or a spatially ordered state. Their size is easily tunable in the range $R=0.5-15 \mu \mathrm{m}$. Whereas the shear modulus of the amorphous lamellar foam is alike that of usual liquid foams or concentrated emulsions and scales linearly with $1 / R$, the elastic modulus of the ordered foam is almost independent of the cell size. This result — probably the first describing the elasticity of an ordered foam-like system- remains unexplained.
\end{abstract}

PACS. 62.20.Dc Elasticity, elastic constants - 83.50.Fc Linear viscoelasticity - 83.70.Jr Liquid crystals; nematic, cholesteric, smectic, discotic, etc.

\section{Introduction}

Determining the elasticity of a material is probably one of the first steps in the characterization of its mechanical properties. In the frame of linear response theory, elastic moduli are closely related to the equilibrium properties of the material. A fair description of the latter, mainly through an adequate free-energy writing and a good knowledge of the structural properties, is required for that approach. It has been used to date in various scientific areas such as the study of sound propagation in crystals, 2nd sound in liquid crystals, the dynamic response of "living" micelles, etc. In the case of "soft" matter, the interest is often centred around the so-called shear modulus $G^{\prime}$, as defined in linear viscoelastic experiments. The aim is then to relate its behaviour as a function of frequency, temperature, concentration, etc. to structural parameters and interactions defined at the mesoscopic length scales characteristic of such materials. As soon as the material is not at thermal equilibrium, and apart from the experimental ambiguities that may then arise, theoretical grounds of elasticity have to be questioned. This includes in particular the role of disorder and frustration. It appears to be crucial, as recently addressed in a theory describing the rheology of "soft glassy materials" [1]. Based upon the common viscoelastic behaviour of a large class of soft materials (mainly elastic with a wide spectrum of dissipation), the model assumes that those materials are metastable and disordered. The mechanical response of the

\footnotetext{
a Present address: Department of Physics \& Astronomy, The University of Edinburgh, The Kings' Buildings-JCMB, Mayfield Road, Edinburgh EH9 3JZ, UK.

e-mail: leng@ph.ed.ac.uk
}

material is described through the intrinsic reorganisation dynamics at a mesoscopic scale. This situation is well exemplified by emulsions: by increasing the volume fraction of droplets, the material gains a (specific) shear modulus above the random packing volume fraction $\phi_{0}$ but is still able to flow through the reorganisation of "clusters" of particles. Oppositely, pioneering models of emulsion elasticity were based on periodic arrays of cells [2] but failed to describe the smooth increase of the elastic modulus $G^{\prime}$ above $\phi_{0}$ [3]. It is now established that the modulus $G^{\prime}(\phi)$ is correctly described only by invoking both disorder and peculiar interactions between droplets [4].

In this article, we present the elastic properties of a lamellar liquid foam as a function of the spatial organisation of the droplets. The system consists of multilamellar vesicles (the so-called onion texture) as generated by a simple shear flow on a lyotropic lamellar phase [5-8]. These vesicles fill up completely the space (volume fraction $\phi \approx 1$ ) and their size is determined by the shear rate [6]. It has already been shown that the elastic response of this material is similar to emulsions: It may be described by a kind of surface tension, expressed in terms of the elastic constants of the native lamellar phase, but includes in addition the contribution of disorder [9]. By using the ability to swell the lamellar phase (and therefore to change the stiffness of the lamellar order), it was possible to tune the effective surface tension and to find a good agreement with the theoretical model [9]. With the system we study here, we take advantage of the so-called layering transition that occurs at intermediate shear rates in a finite temperature range $[10-12,8]$ to gain some control over the spatial organisation of the onions, additionally increasing the range of their sizes. As explained in 


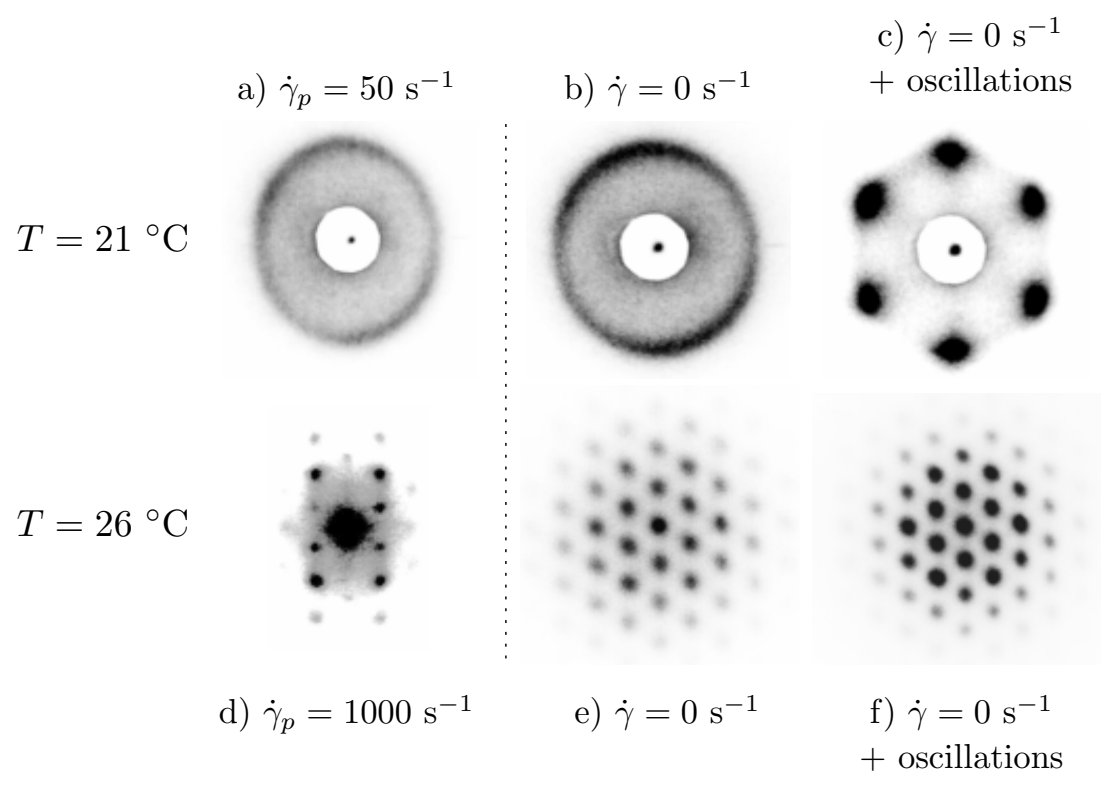

Fig. 1. Examples of SALS patterns of shear-ordered structures of small onions (top) and big onions (bottom). The spatial correlation of the onions is not always visible under shear (a vs. d) and usually does not persist once the shear is stopped. It is however possible to induce it again at rest by acting oscillations by hand on the rotor of the Couette cell (c,f).

more detail elsewhere [12], it is possible to prepare longrange-ordered onion states with quite a long lifetime in addition to the more common, disordered (or amorphous) state. We are thus able to compare the linear viscoelastic properties for the two different spatial organisations. Though the frequency dependences of the shear and loss moduli are qualitatively similar for both the ordered and amorphous states, the dependences on the onion size $R$ differ markedly, with a plateau value for $G^{\prime}$ displaying little variation with $R$ in the ordered state. Our results are probably the first experimental test of the elastic properties on an ordered foam-like system and, as we shall see, they open fundamental questions. In particular, the role of the disorder remains to be understood.

\section{Effect of shear on lyotropic lamellar phases}

The effect of shear on lyotropic lamellar $\left(L_{\alpha}\right)$ phases has been widely studied and it is now well known that the orientation of the surfactant bilayers is extremely sensitive to shear flow. We focus our attention on the onion texture often encountered in an intermediate range of shear rate. This texture is made up multilamellar and closepacked vesicles [6] of a monodisperse size $R$ which is, to a large extent, fixed by the shear rate $\left(R \propto \dot{\gamma}^{-1 / 2}\right.$, laying in the range $0.1-10 \mu \mathrm{m})$. Therefore, small-angle light scattering technique (SALS) is a very convenient means to probe the onion state. A typical SALS pattern consists of a relatively thin ring (Fig. 1a) which not only shows that the size is uniform and well defined throughout the sample, but also reveals that there is no long-range order in the onion positions. Further experiments have shown that the structure is probably liquid-like or amorphous,

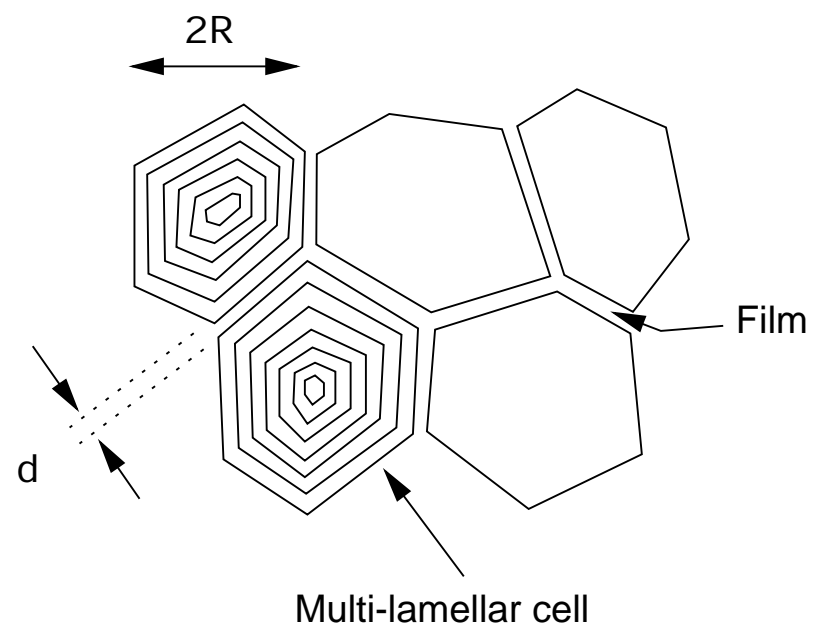

Fig. 2. Schematic representation of the onion texture as a lamellar foam: each cell is a multilamellar vesicle of polyhedral shape and cells are separated by a solvent film of thickness $d$, the smectic distance. This implies a volume fraction $\phi \approx 1$.

with a local order that does not extend further than typically the third neighbour. In such a stacking and because of the high filling ratio, onions are deformed and adopt a polyhedral shape whose exact geometry depends upon the stacking [13]. This point motivates a structural analogy with dry foams as illustrated in Figure 2. The volume fraction $\phi$ occupied by the vesicles is necessarily very close to 1 . Indeed, the film separating the cells is nothing but a normal inter-bilayer space of thickness $d$, the smectic distance. Based on a spherical geometry, a crude estimate of 
the volume fraction reads:

$$
\phi \approx \frac{R^{3}}{(R+d / 2)^{3}} \approx 1-\frac{3}{2} \frac{d}{R}
$$

since $d \ll R$. We get $\phi \approx 0.94-0.99$ for typical values of $R$ and $d$ and the regime is therefore that of $d r y$ foams. Note additionally that this volume fraction is presumably nearly insensitive to the type of packing.

We now describe a protocol to generate an ordered onion texture. The corresponding lamellar phase is a made of sodium dodecylsulphate, octanol and brine (respectively, $7,8,85 \%$ in weight with $20 \mathrm{~g} / \mathrm{l}$ of $\mathrm{NaCl}$ in water, in the present study - see Ref. [14] for a phase diagram). Under a steady shear flow, the behaviour is strongly temperature sensitive and a shear diagram in the plane $(T, \dot{\gamma})$ has been built [11]. In addition to the three stationary states usually encountered (oriented membranes-onionshighly oriented membranes), two other states were observed. They correspond also to onion textures, but differ from the former by their spatial organisation. Above a characteristic shear rate $\left(\dot{\gamma} \approx 50 \mathrm{~s}^{-1}\right.$, somewhat temperature dependent), one observes a clear evolution in the SALS pattern, with six lobes symmetrically superimposed to the ring observed at lower shear rates [10]. This structural transition was referred to as the layering effect, in analogy with the shear ordering of colloidal suspensions: the onions are located on hexagonal planes slipping past each other under flow [10]. Further increasing the shear rate, the size of the onions, though still decreasing, does not change much until a new transition takes place. The characteristic shear rate, and even the very nature of this transition, both strongly depend on the temperature. At low temperature $\left(T<25{ }^{\circ} \mathrm{C}\right)$, the oriented state with bilayers parallel to the flow is recovered (onion-breaking transition), whereas at high temperature $\left(25<T<30^{\circ} \mathrm{C}\right)$ a jump in the size of the onions is observed (jump-of-size transition). This new onion state is made of large onions again ( $R$ typically 10 to $20 \mu \mathrm{m}$ ), and it is also well ordered under shear [11], see Fig. 1d). Among the remarkable features of these new onion states, there is the possibility to reach a state of well-ordered onions, not only under shear but also at rest. Whereas the shear-induced order beyond the layering transition does not always persist once the shear is stopped (see Fig. 1b), one may induce it again by acting oscillations by hand on the rotor of the shear cell (with a strain of a magnitude $\gamma \approx 20-40 \%$ at a frequency $f \approx 10 \mathrm{~Hz}$ ). This causes the SALS pattern to become extremely anisotropic, as shown in Fig. 1c). The same result also obtains in the high-temperature ordered state, which anyway remains ordered when shear is stopped. These small oscillations then obviously improve the sharpness of the SALS patterns, compare Fig. 1e) and 1f). Moreover, these SALS patterns are left unaltered by a quench of the sample at rest to temperatures lower than $25^{\circ} \mathrm{C}$. By this means, and depending upon the experimental parameters during the preparation process (stationary flow at temperature $T$ and under shear rate $\dot{\gamma}$ ), one generates a sample at rest and low temperature of either small ordered onions $(R \approx 0.3-5 \mu \mathrm{m})$ or big onions $(R \approx 10-20 \mu \mathrm{m})$ with a

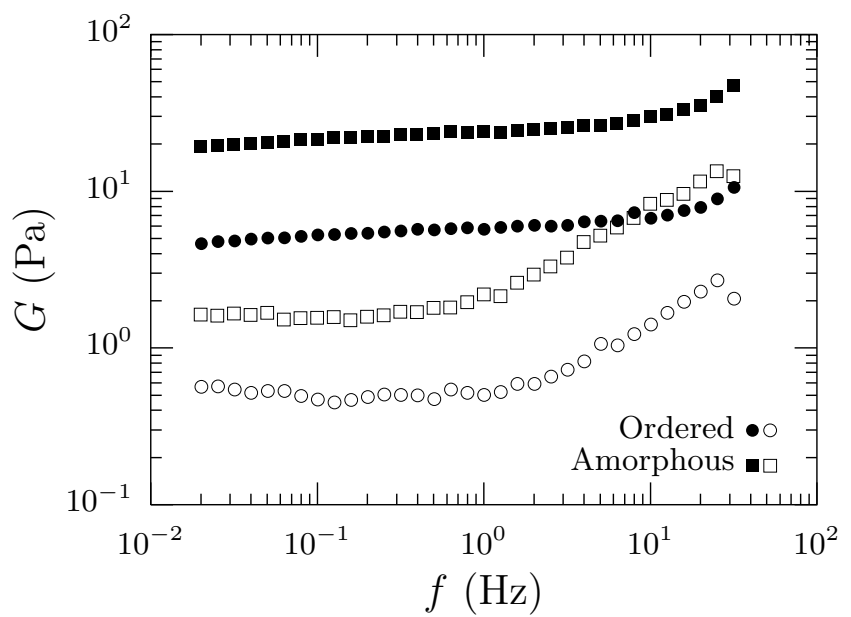

Fig. 3. Linear viscoelastic response for the two organisations of onion samples as a function of frequency. In both the amorphous and ordered states, the onion radius is $R \approx 0.5 \mu \mathrm{m}$, corresponding to onions prepared below the jump-in-size transition. (Filled symbols are for the shear modulus $G^{\prime}$, open symbols for the loss modulus $G^{\prime \prime}$.)

remarkable long-range order. In the latter case, the structure has been characterised using SALS and small-angle neutron scattering [12]. It consists in the regular stacking of hexagonal planes without long-range correlations of the phase sequence, usually referred to as a random close packing. This ordered structure is usefully contrasted to the amorphous one already mentioned. The latter may be obtained along several different paths in the orientation diagram: simply shearing the sample at a relatively low shear rate (until a steady state is reached) and stopping the flow abruptly leads to large onions $(R \approx 5-10 \mu \mathrm{m})$. Alternatively, one can pass through the layering transition to obtain smaller onions. As mentioned previously, the order generated under flow may be lost at rest: an amorphous state of small onions $(R \approx 0.5-3 \mu \mathrm{m})$ is reached, with SALS patterns, Fig. 1b), displaying no evidence of any long-range order. Finally, the ordered state of large onions prepared at high temperatures, and still observed at rest and low temperatures, may be destroyed by acting by hand irregular though gentle movements on the rotor of the shear cell: an amorphous state of large onions $(R \approx 10-20 \mu \mathrm{m})$ obtains in this latter case.

\section{Viscoelastic investigation}

Based on these two structures observed over a wide range of onion sizes, we have conducted viscoelastic measurements in the linear regime. Onions are prepared by shearing the lamellar phase until a steady state is reached (as established by the stationarity of the viscosity). To control the organisation of the ordered texture, we use a transparent Couette cell mounted on a rheometer, coupled with SALS. We use either a stress-controlled (Carrimed CSL100) or a strain-controlled (Rheometrics RFS2) 
rheometer. The results do not depend on the apparatus nor on the geometry of the shear cell. We thus confidently assume that no wall-slip affects the measurements. The reproducibility is tested over a large number of experiments. For each onion size, the strain extent of the linear regime is measured at frequencies $f=0.1$ and $1 \mathrm{~Hz}$. The measured linearity is not noticeably affected by $f$, although the limit $G(\gamma \rightarrow 0)$ may vary slightly with it. We find that the strain should not exceed $\gamma \approx 0.5 \%$ for the largest onions whatever the spatial organisation. Note, however, that in the ordered case the determination of the linear domain may alter the structure. In order to prevent this bias, we first measure the extent of the linear domain for each onion size and then again go along the previously described path in the orientation diagram to restore the best possible ordered texture at rest. Because the whole procedure is quite time-consuming, in many experiments the extent of the linear regime is estimated rather than truly measured. Nevertheless, such ancillary studies are a pre-requisite for careful investigations.

A representative result for the frequency dependence of the elastic moduli $G^{\prime}(f)$ and $G^{\prime \prime}(f)$ is displayed in Figure 3 , for small $(R \approx 0.5 \mu \mathrm{m})$, and ordered as well as amorphous onions prepared below the jump-in-size transition. The frequency dependences are analogous in both cases, the magnitude only of the moduli being affected by the spatial order. The shear modulus $G^{\prime}$ is almost constant at low frequencies and characterised by a "plateau" value $G_{\mathrm{p}}^{\prime}$, measured at $1 \mathrm{~Hz}$ [9]. The onset of a high-frequency rise, not really accessible in our experiment, may be guessed or observed in our data. The loss modulus $G^{\prime \prime}$ exhibits a shallow minimum, followed by a high-frequency rise. Because $G^{\prime \prime}$ is an odd function of the frequency, it must vanish at $f=0$. The broad minimum at $f \approx 0.1 \mathrm{~Hz}$ reflects the presence of a wide spectrum of dissipative processes at even lower frequencies. Our experimental frequency window is therefore located just in between two dissipation modes which cannot fully be appreciated in classical rheology.

We now focus our attention on the shear modulus of these lamellar foams. Figure 3 clearly shows that the spatial organisation of the onions strongly affects the magnitude of the moduli. Indeed, except for the long-range ordering of the onions, everything in the material is similar. The ordered structure is here approximatively three times "softer" than the amorphous texture. We show below how this ratio depends on the onion size. Figure 4 is a plot of the plateau value $G_{\mathrm{p}}^{\prime}$ of the shear modulus as a function of the reciprocal size $1 / R$ of the onions, for the two structures. Whereas the modulus of the amorphous texture varies strongly, approximately linearly with $1 / R$, the ordered structure exhibits a modulus nearly independent of the size.

\section{Discussion}

The size dependence of $G_{\mathrm{p}}^{\prime}$ is apparently in contradiction with a previously developed model [9]. It is argued in this model that the elasticity of the amorphous texture of onions comes from two contributions. The first

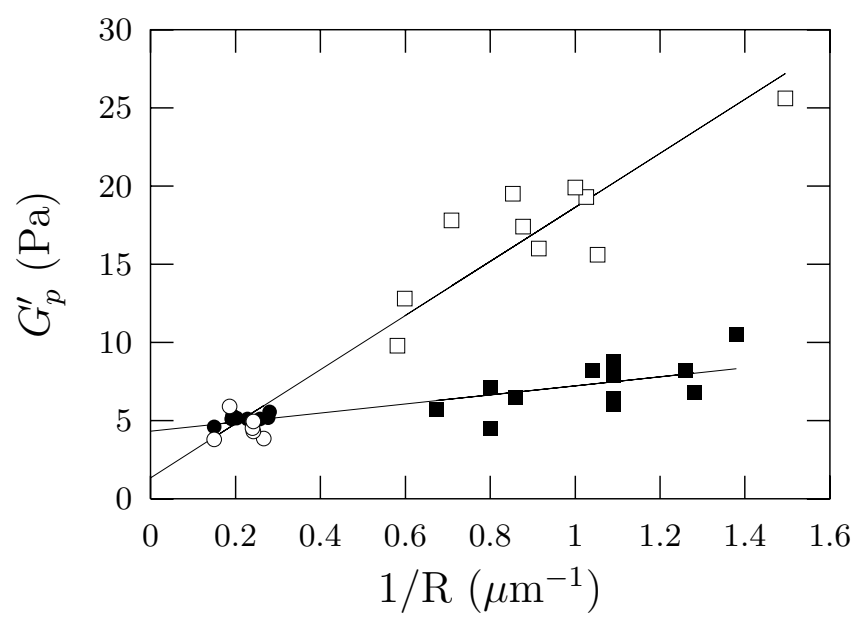

Fig. 4. Plateau value of the shear modulus $G_{\mathrm{p}}^{\prime}$ as a function of the reciprocal onion size $1 / R$, for ordered (full symbols) and amorphous (open symbols) structures. Squares stand for small onions prepared above the layering, but below the jump-in-size transition. Circles stand for large onions, either prepared at low shear rates below the layering transition (amorphous state) or beyond the jump-in-size transition (ordered state).

one originates from the energetical cost of deforming an onion. As any other smectic A liquid crystalline phase, the $L_{\alpha}$ phase is characterised by two elastic moduli, one for the compression of the smectic layers $\bar{B}$, the second $K$ for bending the layers [15]. The combination $\sqrt{K \bar{B}}$ is of a special interest since it has the dimensions of a surface tension and, indeed, plays the role of an effective surface tension for small deformations of lamellar structures [15]. This result is strictly true for a spherical lamellar object [16], and qualitatively valid for polyhedral ones [9]. Consequently, the first contribution to the shear modulus scales as $G_{\mathrm{p}}^{\prime} \sim \sigma / R$, where $\sigma \approx \sqrt{K \bar{B}}$ (this scaling is precisely the one observed in concentrated emulsions, $\sigma$ being in this context the oil-water surface tension [4]). Note that for systems stabilised by steric interactions, $K \bar{B} \sim 1 / d^{4}$ and is not expected to be system dependent. Indeed, for the various previously studied systems [9], the slope of $G_{\mathrm{p}}^{\prime}$ vs. $1 / R$ is in reasonable quantitative agreement with the calculated value of the effective surface tension using values of $K$ and $\bar{B}$ measured independently. Then, it is argued in reference [9] that the measured shear modulus also reflects the quenched disorder in an amorphous structure. The disorder comes from a frustration of the $L_{\alpha}$ lamellae in randomly distorded onions compared to symmetric onions. The quenched or additional strain $\gamma_{0}$ interacts in a complicated manner with an applied strain $\gamma$ to give a contribution to the shear modulus $\alpha \approx \gamma_{0}^{2} \bar{B}$, independent of the size of the onions. This contribution has been observed to be quite pronounced for several $L_{\alpha}$ phases [9]. It has also been experimentally shown that $\alpha$ obeys the scaling law $\alpha \sim 1 / d^{3}$ as expected $\left(\alpha \propto \bar{B} \sim 1 / d^{3}[17]\right)$ if one assumes that $\gamma_{0}$ does not depend on $d$ [9]. In the present case, the relevance of such a contribution is not absolutely clear in Figure 4 (open symbols), since it is only 
revealed by a modest finite intercept at infinite onion size. As a possible explanation for this behaviour, note that $\bar{B}$ is here small $(\bar{B} \approx 300 \mathrm{~Pa})$ and $\alpha$ is accordingly small.

With the simple (maybe incorrect) scheme of polycrystalline domains for the amorphous texture, the shear modulus originates, on the one hand, from the elastic cost of deforming onions inside domains and, on the other hand, from the contribution of the grain boundaries between different domains. The first part scales as $1 / R$ whereas the second one is independent of the onion size and increases with disorder. Consequently, one expects that $\alpha$ vanishes for an ordered foam of onions (where the grain boundaries have disappeared). We see in Figure 4 that we observe something quite different: For the ordered states, $G_{\mathrm{p}}^{\prime}$ is almost independent of the size $R$ and exhibits a non-zero value when $1 / R \rightarrow 0$. This behaviour is surprising and still unexplained.

One may invoke various reasons for explaining this result. First, the elasticity of the ordered structure should be treated through a strict tensorial formalism rather than a scalar one. It might be possible to find peculiar directions for which the shear modulus is a weak function of the size of the droplets. Nevertheless, one easily shows that the direction of strain within a hexagonal layer (in cubic or hexagonal symmetries) does not induce any effect on the stress response. Moreover, this does not explain why $G_{\mathrm{p}}^{\prime}(1 / R \rightarrow 0) \neq 0$. Second, the structure of interest to us is not long-range-ordered stricto sensu. Indeed, it consists of a random sequence of two structures (namely face-centered cubic and hexagonal compact [12]) possessing a priori different elastic moduli. This effect may also induce a particular elastic response but we expect to keep the scaling $G^{\prime} \sim 1 / R$. In addition, it is worth mentioning that the elastic response is measured in the very direction of the flow used to prepare the onions: The stacking faults are consequently perpendicular to the strain direction. Note that "anomalous" elasticities are quite common in heterogeneous solids. Such anomalies are referred to as the "supermodulus" effect, often encountered in layered systems of metallic crystals. These systems are made of a regular sequence of (chemically) different layers. A softening of the shear modulus is observed as a function of the thickness of the layers $[18,19]$. Although still not understood, one reason is often invoked and concerns the role of the compatibility stress which originates at the interface between two different materials.

\section{Conclusion}

We presented the elastic properties of an ordered lamellar foam. This peculiar organisation of soft multilamellar vesicles possesses an elastic shear modulus which is (almost) independent of the foam characteristic size. This result is surprising and cannot be simply explained with the models previously elaborated to describe the elasticity of foams or cellular materials. Although various reasons may be invoked to explain this behaviour, none of these offers a simple representation of the elasticity of our well-ordered system. Therefore, this experimental result remains unexplained but opens the question of a more indepth description of the role of disorder.

\section{References}

1. P. Sollich, F. Lequeux, P. Hébraud, M.E. Cates, Phys. Rev. Lett. 78, 2020 (1997).

2. J.C. Moser H.M. Princen, M.P. Aronson, J. Colloid Interface Sci. 75, 246 (1980).

3. T.G. Mason, J. Bibette, D.A. Weitz, Phys. Rev. Lett. 75, 2051 (1995).

4. T.G. Mason, M.D. Lacasse, G.S. Grest, D. Levine, J. Bibette, D.A. Weitz, Phys. Rev. E 56, 3150 (1997).

5. O. Diat, D. Roux, J. Phys. II 3, 9 (1993).

6. O. Diat, D. Roux, F. Nallet, J. Phys. II 3, 1427 (1993).

7. J. Lauger, R. Weigel, K. Berger, K. Hiltrop, W. Richtering, J. Colloid Interface Sci. 181, 521 (1996).

8. J. Penfold, E. Staples, I. Tucker, G.J.T. Tiddy, A.K. Lodi, J. Appl. Cryst. 30, 744 (1997).

9. P. Panizza, D. Roux, V. Vuillaume, C.-Y.D. Lu, M. E. Cates, Langmuir 12, 248 (1996).

10. O. Diat, D. Roux, F. Nallet, Phys. Rev. E 51, 3296 (1995).

11. P. Sierro, D. Roux, Phys. Rev. Lett. 78, 1496 (1997).

12. J. Leng, P. Sierro, F. Nallet, D. Roux, O. Diat, Structure of a shear-ordered lamellar phase, in preparation.

13. T. Gulik-Krzywicki, Electronic microscopy pictures of onions, private communication.

14. P. Hervé, D. Roux, A.M. Bellocq, F. Nallet, T. GulikKrzywicki, J. Phys. II 3, 1255 (1993).

15. P.-G. de Gennes, J. Prost, Physics of Liquid Crystals (Clarendon Press, 1976).

16. E. van der Linden, J.H.M. Dröge, Physica A 193, 439 (1993).

17. Helfrich W., Z. Naturforsch. A 33, 305 (1978).

18. B.W. Dodson, Phys. Rev. Lett. 60, 2288 (1988).

19. B. W. Dodson, Phys. Rev. B 37, 727 (1988). 